\title{
Autonomía, determinación, razón y valores en las concepciones antropológicas de la teoría organizacional clásica ${ }^{1}$
}

\author{
Gerardo Romo Morales \\ Universidad de Guadalajara \\ gerardo.romo@gmail.com \\ Sarabi Lay-Trigo \\ Universidad de Guadalajara \\ zavlay@hotmail.com
}

\begin{abstract}
Resumen: El presente artículo responde a una indagación importante para la sociología y la filosofía aplicada a un espacio concreto del campo cientifico. El cuestionamiento es: ¿hay en la teoría organizacional (TO) una concepción antropológica -explícita o implícita- relacionada con las duplas conceptuales de autonomía-determinación y razón-valores? La respuesta se elabora a partir de una revisión de las concepciones antropológicas y ontológicas que están presentes en los discursos científicos y en las propuestas teóricas de los autores clásicos de esta disciplina. Con ello develamos sus posturas frente al ser/sujeto/individuo/actor/agente que participa dentro de las organizaciones humanas y buscamos abonar el entendimiento de sus capacidades, habilidades y atribuciones otorgadas dentro del espacio de convivencia socioorganizacional. Palabras clave: teoría organizacional; concepciones antropológicas; autonomía-determinación; razón-valores.
\end{abstract}

\footnotetext{
1 Este artículo contó con la colaboración, en calidad de asistentes, de los siguientes estudiantes miembros del semillero de investigación «Grupos, organizaciones, instituciones y políticas públicas»: Carmen Ramos Godínez, Ricardo Ávalos Sotelo, Óscar Jiménez Sánchez, Kimberly Lazos Zubía.
} 
Autonomy, determination, reason and values in the anthropological conceptions of classical organizational theory

\begin{abstract}
The present article addresses an important question for sociology and philosophy applied to a specific space in the scientific field, which is the following: is there within Organizational Theory (OT) an explicit or implicit anthropological conception related to the conceptual pairs of Autonomy-Determination, and ReasonValues? The answer is based on the anthropological and ontological conceptions that are present in the scientific discourses and theoretical proposals of the classic authors of this discipline. With this we aim to understand what are their positions towards the being / subject / individual / actor / agent that participates within buman organizations, as well as to understand what are the capacities, abilities, attributions that they give to them within the space of social-organizational coexistence.
\end{abstract}

Keywords: organizational theory; anthropological conceptions; scope and limits of OT. 


\section{Introducción}

Para el presente artículo partimos del supuesto de que todo corpus teórico se posiciona ontológicamente frente a la realidad, es decir, que todo autor tiene una postura sobre el ser humano con la cual le atribuye ciertas características y que, por lo tanto, esta puede ser reflexionada desde la filosofía ${ }^{2}$ y la teoría social. El artículo está dividido en cuatro apartados. En el primero hablamos de los autores clásicos de la TO a partir de una definición elaborada ex profeso y señalamos quiénes son, mostrando los criterios que utilizamos para su selección. En el segundo apartado, hacemos una reflexión sobre lo que son las concepciones antropológicas, a través de un breve recorrido por el pensamiento filosófico que nos permite definir lo que entendemos por esta importante categoría. En el tercer apartado relacionamos a los autores y escuelas clásicas de la TO con las concepciones antropológicas, es decir, reunimos en un argumento lo que habíamos elaborado en los dos apartados anteriores. Y finalmente, con todos estos antecedentes, en el cuarto apartado nos detenemos en las diferencias específicas de los autores con respecto a las categorías concretas de nuestro interés, y las relacionamos con la discusión con la que consideramos se corresponden en las ciencias sociales y que hemos reunido en las duplas autonomías vs. determinación y razón $v s$. valores o normas.

\section{La definición de lo clásico y los autores de la TO}

Partimos de la definición hegeliana que señala que el arte deviene clásico cuando se convierte en ideal y logra interpretarse a sí mismo perdurando a lo largo del tiempo. Esta idea la consolidamos con la de Gadamer, para quien lo clásico es aquello que no solo cumple la condición previa, sino que además se mantiene «frente a la crítica histórica porque su dominio histórico, el poder vinculante de su validez transmitida y conservada, va por delante de toda reflexión histórica y se mantiene en medio de ésta» (Gadamer, 1999: 356).

Si Gadamer refuerza la idea de perdurabilidad para la comprensión de lo clásico, citamos a continuación a dos teóricos que le añaden la condición de ideal, de referencia y, por lo tanto, de modelo legítimo a imitar. Así es cómo el «adjetivo clásico existía en el siglo xviı, para calificar un trabajo que merecía ser imitado, servir como modelo, ser considerado una autoridad» (Compagnon y Cosman,

2 Entendemos la filosofía como una disposición, una actitud ante el conocimiento, «un compromiso activo con la verdad. Es en este sentido en el que debe ser entendida la traducción habitual del término philosophia como “amor al saber" o"a la sabiduría” (Fernández, 2010: 12). 
2004: 177) ${ }^{3}$. Un argumento más que se encuentra en la literatura para definir lo clásico, y que de alguna manera complementa lo anterior, es su carácter funcional y de actualidad, el cual incorpora al respecto el filólogo Jauss. Para este, «[... ] la oposición histórica entre lo romántico y lo clásico se reduce a la oposición relativa entre lo que para los contemporáneos es actual, pero para la siguiente generación vuelve a ser ya algo superado, y [por lo tanto] lo pasado» (Jauss, 2013:65).

A partir de lo anterior podemos expresar una definición operativa de lo clásico. Diremos que algo puede llamarse clásico cuando perdura en el tiempo, se mantiene actual y se reconoce como autoridad, es decir, como modelo o referencia a imitar, y que, por lo tanto, legitima - como criterio de orden en un sentido weberiano - lo que se produce en el campo de referencia. Esta definición es general, y podría incluir autores u obras artísticas o filosóficas y científicas. Pero para estas últimas, tendríamos que agregar el hecho de que sean capaces de explicar fenómenos actuales. Un criterio que permite evidenciar la condición enunciada, con respecto a la condición de autoridad y criterio de legitimación, es el de la repetición en su uso como referencia. Es decir, y solo como ejemplo, los autores clásicos de la literatura latinoamericana, serán aquellos que más se lean, más se emulen, y más se citen. Los compositores clásicos de la música de orquesta serán los que más se estudien como modelo y más se interpreten. Pero en una ciencia como la biología, la física o la sociología, las obras clásicas serán, igualmente, las más citadas o referidas.

La condición de clásico no significa univocidad o hegemonía diacrónica, ni en el arte ni en la ciencia. En esta última, cuando uno mira hacia atrás en cualquier disciplina, va a ser capaz de encontrar diferentes representantes así adjetivados, representando épocas que se constituyen a sí mismas como sistemas de acción concreto. Esto permite, en orden consecutivo o bien sincrónico, pero siempre ex post, reconocer, distinguir y analizar diferentes enfoques o posiciones iniciales de abordaje de los temas en cuestión, es decir, la naturaleza teórico-metodológica, epistemológica, ontológica, antropológica, que cada autor propone para estudiar la realidad. De esta manera, en lo que sigue vamos a aplicar los criterios anteriores para señalar a los autores clásicos de la disciplina como aquellos que en una mayor cantidad de veces son leídos citados y, por lo tanto, utilizados para explicar fenómenos relacionados con las acciones organizadas; y que se presentan de manera secuencial en un período de tiempo determinado. Pero ¿cuáles son esos autores clásicos que serán parte de nuestra reflexión? Para evitar una simple enunciación intuitiva que nos llevaría a generar una lista de autores, aparente-

3 La traducción es nuestra. La cita original dice: «The adjective classic itself existed in the seventeenth century, when it qualified a work that deserved to be imitated, to serve as a model, to be considered authoritative». 
mente indiscutibles como clásicos, nos dimos a la tarea de constatar la existencia de los mismos mediante el recurso y los criterios enunciados antes.

En lo que sigue, presentamos lo que resultó del rastreo que hicimos en la literatura sobre TO y en los programas de las materias que más se utilizan en los cursos universitarios correspondientes. En los cursos formativos sobre TO hay que señalar que existen de manera hegemónica ciertas escuelas y autores que son los más citados, los más referidos y, por lo tanto, los más repetidos. Asumimos entonces que la repetición no es gratuita o inercial, sino que más bien corresponde a que estas teorías y estos autores constituyen las bases fundadoras y paradigmáticas que dan lugar a la continuación, el complemento o la generación crítica de autores y perspectivas modernas y contemporáneas para el estudio de las organizaciones como entidades meso de la teoría social. Así, buscamos la presencia de los autores en la bibliografía especializada en la recolección histórica y teórica del pensamiento organizacional en español y en inglés. Consultamos los textos de Ahumada (2001), Alonso y Ocegueda (2006), Dávila (2001), Ibarra (1999), March y Simon (1980), Pariente (2001), Pugh y Hickson (2007), Ramió (2010), Ramió y Ballart (1992), Rivas (2009), y Shafritz, Ogg y Jang (2015), es decir, textos que, durante más de treinta y cinco años, en diferentes países de habla hispana e inglesa, han marcado una fuerte coincidencia con respecto a cuáles son los autores más analizados y, por lo tanto, según nuestro criterio, los clásicos de la TO.

Encontramos ciento veintiséis autores diferentes que van desde aquellos que escribieron desde los primeros años del siglo xx hasta los autores más contemporáneos del siglo xxi, y entre estos, los más citados son Max Weber (1864-1920) y Frederick Taylor (1856-1915), con diez menciones (los únicos que aparecen en todos los textos revisados); Henri Fayol (1841-1925), con nueve; Elton Mayo (1880-1949) y Herbert Simon (1916-2001), con ocho; con siete, Philip Selznick (1919-2010), Douglas McGregor (1906-1964) y Henry Mintzberg (1939-); con seis, Chris Argyris (1923-2013) y Chester Barnard (1886-1961); y con cinco, Michel Crozier (1922-2013), Rensis Likert (1903-1981), James March (1928), March y Simon, Steward Clegg (1947-), Oliver Williamson (1932-) y Edgar Schein (1928-).

Con respecto a los autores utilizados en los programas formativos de TO en programas de pregrado y posgrado, se revisaron los casos de universidades de distintos países (México, España, Colombia, Argentina, Alemania, Suiza, Estados Unidos y Rumanía). Los programas universitarios analizados pertenecen a las materias de TO de diferentes universidades, como la Universidad de Guadalajara, la Universidad de Almería, la Universidad de la Amazonia, la Universidad Autónoma Metropolitana, la Universidad Nacional del Nordeste, la Universidad 
Técnica de Múnich, la Universidad de Zúrich, la Universidad de Montana, la Universidad de Stanford, y la Universidad de Bolyai. En este caso, los autores más citados fueron Frederick Taylor (1856-1915), con siete referencias, y Max Weber (1864-1920), con cinco; destacan con tres Henri Fayol (1841-1925), Charles Perrow (1925-), Herbert Simon (1916-2001), James March (1928-) y Alfred Chandler (1918-2007); y los textos de Paul DiMaggio (1951) y Walter W. Powell $(1951)^{4}$. Con esto, confirmamos el criterio de repetición que es importante para nuestra definición de clásico.

Al comparar las columnas de referencias en libros y en programas universitarios, nos dimos cuenta de que hay autores que son relevantes para los cursos de la materia de TO que no son necesariamente los más citados en la bibliografía especializada. Esto, más que una contradicción o una debilidad de la disciplina, tendría que ser visto más bien como un factor o elemento complementario. $\mathrm{Pa}$ rece que las escuelas o los principales argumentos son los mismos, aunque en algunos casos se recurra a otros autores, tal vez porque son más didácticos o más conocidos para los profesores de los diferentes departamentos de las diversas universidades. Este sería el caso de Charles Perrow o de Alfred Chandler, con las mismas menciones que Fayol, pero que en la relación de los libros revisados antes solo tiene una mención.

Ahora, además de la repetición, habrá que destacar el criterio de perdurabilidad en los autores con los cinco índices ponderados más altos: podemos ver con claridad que este principio se cumple en la lista anterior cuando observamos el año de publicación de su obra principal, sobre todo con Taylor (1916), Fayol (1916) y Weber (1922), y en menor grado con Mayo (1933) y Simon (1946). De la misma manera, podemos considerar el hecho de que se mantengan las obras siendo actuales a partir de su aparición repetida en los presentes cursos universitarios sobre la materia y, por lo tanto, también es claro que se consideran como modelo o referencia. En este sentido todos tienen un índice alto con excepción de Mayo.

En términos operativos, para poder hacer el análisis en este artículo recurrimos a la integración que se ha hecho en la literatura y los programas de las escuelas que conjuntan a los autores. Entre estas, habrá también en la literatura o en los cursos de formación unos más o menos importantes.

En este sentido, van a ser la escuela de la administración científica y/o clásica, de Taylor y Fayol junto con la del análisis de la burocracia de Weber las escuelas más citadas. Les siguen la de las relaciones humanas con Mayo como máximo representante, y la de las decisiones y el comportamiento con Simon. Esto nos

4 En la lista de los libros, aparece cuatro veces, sumadas las que son de Powell y Dimaggio y las que son de Dimaggio y Powell. 
parece que tampoco es gratuito, en realidad, las dos primeras corresponden a los dos grandes pilares de la teoría organizacional: por una parte, el de la administración, con su carácter normativo y en pro de la eficiencia; y por la otra, el de la sociología, con una finalidad más bien comprensiva o de explicación. Las otras dos escuelas, con la psicología y la economía como disciplinas iniciales, respectivamente, van a complementar una base de cuatro puntos para la robusta y sólida estructura transdisciplinar del pensamiento organizacional. Será con estas escuelas y sus principales representantes con los que trabajaremos.

Con lo señalado en este apartado, el análisis realizado y el índice ponderado de aparición más alto de ciertos autores, confirmamos: habemus clásicos. Autores invariables e ineludibles que a pesar de haber expresado sus pensamientos y teorías a principio de siglo pasado siguen siendo actuales.

\section{Hablar de concepciones antropológicas}

Antes de entrar de lleno en el análisis de nuestros autores, es importante reflexionar sobre los elementos que buscamos en sus concepciones antropológicas. Asumimos que para cada teoría en ciencias sociales hay al menos dos raíces que pueden ser reflexionadas desde la filosofía: una ontológica, es decir, la de las características que se le atribuyen a los seres humanos en sociedad y a la realidad en tanto ser, ¿cómo es la realidad? y ¿qué es el ser humano?, a la cual llamaremos concepción antropológica; y otra epistemológica, referida más bien a los modos en los que se plantea el acercamiento empírico, el sentido y la comprensión de la realidad.

Sin embargo, no buscamos hacer ciencia del hombre en sí, sino más bien abonar esta gran reflexión desde el espacio que se abre al responder a la pregunta ¿cómo conciben al ser humano las principales escuelas de la TO? Lo que vamos a aportar de manera concreta es cómo una teoría — en este caso organizacionalconcibe al ser/sujeto/individuo/actor/agente. Así, lo que se pretende aclarar son las atribuciones, las características que se le otorgan a los seres humanos dentro de la realidad organizacional. Creemos que este ejercicio - el de la comprensión de las raíces antropológicas - es necesario para toda persona que quiera investigar la realidad organizacional, ya que lo dicho sobre los seres que forman y dan vida a las organizaciones - si lo que se quiere es mantener coherencia teóricaha de definir la postura epistemológica con la que el investigador ha de acercarse a su fenómeno de estudio.

Ahora bien, la pregunta ¿qué es el ser humano? ha estado presente a lo largo de la historia de la filosofía, ha sido una preocupación para los filósofos desde el pensamiento griego y, puesto que la filosofía es una interpretación sobre la realidad, las imágenes que se nos presentan sobre el ser son históricas y, como 
tales, corresponden a una etapa específica que tiene antecedentes, posturas y discusiones. Dentro de este horizonte de concepciones antropológicas podemos decir que estas discusiones tienen elementos que funcionan como límite a las aproximaciones. Se ha dicho que el hombre es un ser racional (Descartes); que el hombre trabaja, es histórico y social (Marx); que el hombre es una invención o construcción reciente (Foucault); que el hombre es una dimensión dialógica que termina cuando muere (Heidegger); que el ser humano puede convertirse en el deber ser (Kant); es el ser frente a la felicidad (Aristóteles); es el hombre caracterizado como ente social (Sócrates, Hobbes), como ente político (Platón) o dual (Aristóteles, Santo Tomás); se define como cooperativo y solidario o como individualista y egoísta; como producto material de la evolución de la naturaleza (cuerpo) o como creación divina (San Agustín); está determinado por una serie de posibles elementos estructurales (Marx, Freud), o es libre y autónomo (Sartre); que el hombre es un ser histórico (Hegel) o es un ente económico (Marx), o que el ser es racional y científico (Comte, Descartes). También se ha dicho que el hombre es una máquina que piensa (la ilustración), que es un ser sentimental, emotivo y pasional (empirismo), o que está condicionado por la subjetividad de sus pasiones (el romanticismo); o bien, que la realidad solo puede ser pensada pero no conocida, a partir de que solo tenemos acceso al fenómeno (kantismo).

De esta larga historia de reflexión ontológica tejida como red conceptual que aquí apenas enunciamos, tenemos como continuos posibles para la acción, la decisión y el orden en la realidad humana el de la libertad frente a la determinación y el de la razón frente a los valores.

Una vez expuesto lo que entendemos por concepción antropológica, de mostrar - aunque de manera resumida - cómo a lo largo de la historia es posible reconocer distintas maneras de comprender al ser humano, de atribuirle capacidades, habilidades y limitaciones, en el siguiente apartado vamos a trasladar la discusión al nivel de las escuelas de la TO y sus formas de entender al ser humano cuando lleva a cabo acciones organizadas.

\section{Escuelas teóricas de la TO y sus concepciones antropológicas}

Preguntarse por las concepciones antropológicas de los autores de la TO pretende confirmar —o no- una hipótesis que plantean Crozier y Friedberg (1989) cuando señalan que el hombre de Taylor es una mano, el de la escuela de las relaciones humanas de Mayo, un corazón, y que por lo general se olvida que el hombre, antes que nada, es una cabeza, es decir, que, además de la capacidad física y los sentimientos, habrá que considerar en su comprensión a la razón y la libertad. 
En esta primera aproximación y recurriendo a nuestros continuos podríamos pensar que para la dupla de sociólogos franceses (Crozier y Friedberg) Taylor asume al ser como determinado, tan determinado como lo está una mano sujeta a las decisiones del sistema nervioso; mientras que el ser de Mayo sería un individuo sensible que al llevar a cabo sus acciones atiende/escucha/consulta elementos mucho más valorativos que racionales. $\mathrm{Y}$ sin darse cuenta, podemos ver con claridad que el hombre que sugieren estos autores al hacer la crítica es un ser libre y racional.

Insistimos aquí en que la pregunta con la que se inicia este apartado se enmarca en la vieja discusión de las ciencias sociales en la que sus principales autores proponen distintas teorías y modelos de aproximación para el estudio del ser humano en sociedad (Marx — paradigma del conflicto-; Compte, Nagel y Popper — paradigma positivista —; Dilthey, Weber y Collingwood — paradigma comprensivista -; Habermas — paradigma comunicativo-, por señalar algunos de los más importantes), a partir de considerar dos como los problemas centrales de la reflexión social: el que tiene que ver con la acción («El "problema de la acción" [...] consiste en dar por sentado que los actores son racionales o no») y el del orden («Los sociólogos son sociólogos porque creen que la sociedad respeta patrones, estructuras independientes de los individuos que la componen») (Alexander, 2008: 18).

Con respecto a la concepción del sujeto, la solución que se intente al primer problema (el problema de la acción) es la que se va a considerar en el continuo de lo racional (egoísta, instrumental, en búsqueda de lo eficiente), o lo normativo (soluciones idealistas, normativas o morales, regidas por emociones o deseos inconscientes); mientras que la solución al segundo (el problema del orden) es lo que se relacionaría directamente con nuestro otro continuo: el ser es libre (el orden es producto de la negociación individual, voluntarista) o determinado (explicación colectiva, los patrones son previos a toda acción individual).

Hasta aquí hemos presentado la discusión hablando de ser, ser humano o individuo. Lo hemos hecho de manera intencional, considerando esa la forma más neutra posible para esta parte del trabajo. Al respecto, asumimos que al hablar de individuos, agentes, sujetos o actores se le está dando una carga ideológica por lo que preferimos hacer esto explícito antes de asumir una postura: en nuestra opinión, quien hable de individuos asume que estos son independientes en la medida en que se contraponen conceptualmente al magma de lo social. Mientras sean individuos están atomizados y móviles; por el contrario, quien habla de sujetos está considerando esa sujeción como determinación del elemento al que esté ligado, al «lazo» que le sujeta. Mientras que la noción de actor es una posibilidad 
intermedia que asume la existencia de un guion o libreto que constriñe, pero que deja espacio para la interpretación, la reformulación o generación de versiones «libres» del mismo, e incluso, la improvisación. En este mismo sentido tendríamos que asumir la idea de agente, como aquel que tiene la capacidad de agencia. Ahora bien, ¿cuáles son entonces las concepciones antropológicas existentes en las escuelas de la TO?

\subsection{Frederick Taylor. Escuela de la administración científica}

En el pasado, el primer lugar le ha correspondido al hombre; en el futuro, el primer lugar debe ocuparlo el sistema (Taylor, 1967: 16).

En la literatura sobre administración y organizaciones hay un consenso en el sentido de que el padre de la administración científica es el ingeniero Frederick W. Taylor, quien desarrolló sus actividades de observación y sistematización de sus resultados en Estados Unidos a finales del siglo xix y principios del xx con el propósito de aumentar la productividad y alcanzar la eficiencia de las empresas. Esta actividad estuvo guiada bajo el principio ideológico de que la máxima prosperidad de cualquiera de los involucrados en una organización significa la máxima prosperidad de los otros (Taylor, 1967). En términos metodológicos el énfasis de sus investigaciones estuvo puesto en los procesos, concretamente en la combinación ideal de «tiempos y movimientos» requeridos para alcanzar ese óptimo de satisfacción y eficiencia. Esa propuesta aparentemente simple de revisión sistemática de los procesos, junto con la de separar el proceso productivo del diseño del mismo, lo cual había permanecido históricamente unido, fue lo que dio lugar al famoso thinking department. Esta separación es la responsable operativa de la enajenación del trabajador, el cual, desde entonces, elabora mercancías de las que no participa en el proceso de imaginación y diseño, ni de la pieza, ni del proceso de elaboración (esto sin considerar que lo que su trabajo produce tampoco le pertenece, perspectiva desde la que el marxismo analizó la enajenación o proceso de fetichismo de la mercancía).

Así, es la combinación de las dos anteriores ideas de Taylor la que permite asumir que para él, y la escuela que representa, el ser, el hombre, es solo una parte mecánica sujeta a lo que el diseño de su rol y actividad le indican. Por lo tanto, no es que el hombre sea libre con toda propiedad, sino que está sujeto. El ser depende en sus acciones del diseño elaborado por otros. Pero entonces, estos otros, ellos, ¿sí son libres? Diremos que tampoco, porque lo único que harán, según Taylor, es seguir la racionalidad de sus decisiones bajo los procedimientos y métodos que la ciencia establece como la única mejor manera de hacer las cosas. Así, el 
one best way es el que cierra el círculo de la comprensión tayloriana del ser, el cual será, en realidad, sujeto de la racionalidad. Este es entonces solo parte de una gran maquinaria, de un gran engranaje social relacionado con la producción. Caracterizamos entonces a esta propuesta como un modelo máquina para el estudio del hombre dentro de las organizaciones. A continuación, desarrollamos esta idea.

Como señalábamos, para Taylor, toda administración debe buscar a través del uso del método científico una prosperidad general y permanente que se construya en común: patrones y trabajadores. Esta prosperidad será también de la empresa y de todas las ramas de la misma en términos económicos y de satisfacción, y se consigue en la medida en que la labor/tarea llegue al óptimo de eficiencia. Como idea general, es posible señalar que gracias a los estudios de la administración científica y su modo de entender la realidad organizacional se comenzaron a observar, medir y desentrañar las jornadas laborales diarias en la empresa. Esto permitió que se perfeccionaran o se encontraran nuevas formas de optimizar la producción. El supuesto de base de Taylor es que los empleadores buscan bajo costo en la mano de obra y los empleados, altos salarios. Así, para encontrar el justo medio era necesario tomar en cuenta cuatro elementos. A saber:

1. Establecimiento de reglas y utilización de la ciencia como herramienta para la estandarización de instrumentos y condiciones de trabajo.

2. Adiestramiento y selección cuidadosa de los trabajadores. Esto incluye eliminar a todos los trabajadores que sean incapaces o que se resistan a adoptar mejores maneras de trabajar.

3. Establecimiento de vigilancia a través de la dirección para mejorar el rendimiento, así como incorporar un sistema de retribuciones que premien el desempeño.

4. Correcta división del trabajo y responsabilidades entre empleados y directores sin olvidar que la dirección debe trabajar codo a codo junto con los trabajadores.

Si se seguían estos pasos era posible asegurar la cooperación de empleados y empleadores para alcanzar la prosperidad y el rendimiento máximo, ya que esto dotaba a la empresa de un orden claro que definía condiciones laborales, planificación, especialización, ejecución, preparación, control, división y estandarización del trabajo, sistema de pagos, beneficios, selección y adiestramiento correcto de los empleados, y se superaba con ello la condición de bajo rendimiento tanto natural como sistemático de los trabajadores. A través de la observación y la medición se podía desarrollar una correcta vigilancia que asegurara el funcionamiento de la organización. Si algo no permitía el óptimo desarrollo de la empresa simplemente tenía que ser removido sin importar si era un instrumento, una téc- 
nica o un trabajador. Desde esta visión el hombre no es más que una pieza de una maquinaria, una mano que facilita el correcto funcionamiento y que se mueve en función de una sola motivación: alcanzar la prosperidad económica.

Para el momento histórico en el que se llevaron a cabo estos estudios y tomando en cuenta la disciplina de formación de Taylor es comprensible - hasta cierto punto- que este autor tuviera una visión mecanicista de la organización en la que el hombre es una mano, un elemento «material» y no humano. El hecho de que los supuestos epistemológicos estuvieran focalizados en modelar funciones, diseñar, dirigir y controlar actividades pudo haber provocado que se perdiera de vista el todo: el ser humano es más que una mano. Por lo que esta postura ofrece una visión deshumanizante, racional, fría, que coloca en el centro de la organización a un ser humano desvinculado, un hombre ejecutor, el homo mecanicus.

Desde nuestra perspectiva este modelo se olvida de dos cosas importantes. Por un lado, que el hombre es más que una parte material dentro de una acción organizada, el hombre es un todo integral: sus emociones, sus relaciones, sus decisiones, sus acciones y sus pensamientos. Por otro lado, entender una organización como una estructura mecánica supone la idea de un equilibrio, o de un supuesto equilibrio, de una constante que sigue su funcionamiento en la medida en que sus partes se dediquen a hacer lo que les toca hacer, en ese sentido si cada uno de sus elementos (materiales y humanos) hace lo necesario, la realidad de la que forman parte debería permanecer siempre igual, como si fuera la maquinaria de un reloj. Sin embargo, sabemos que esto no es así, la realidad es inestable, cambia, se transforma. Hablar de la realidad social como si fuera una máquina es olvidarse de que la acción social es más que una ejecución perfecta, por ejemplo, desde la perspectiva weberiana una acción social es — sobre todo- una acción con sentido.

\subsection{Max Weber. Teoría de la burocracia}

El que ordena obedece, al emitir una orden, a una regla: a la ley o al reglamento de una norma formalmente abstracta (Weber, 1964: 707).

Para Max Weber la acción social puede ser comprendida a través de la interpretación. Este tipo específico de acción será aquella «[...] en donde el sentido mentado por su sujeto está referido a la conducta de otros, orientándose por ésta en su desarrollo» (1964: 5). De esta manera, para este sociólogo alemán, el hombre será «el límite y el único portador del comportamiento provisto de sentido» (Weber, 1964: 187). Este sentido podrá ser racional (actual de pensamientos)o irracional (actual de sentimientos). La sociología de Weber, entonces, se centra 
en las acciones sociales, y estas se explican a partir de la comprensión del sentido mentado por los actores de las mismas. Por lo tanto, su propuesta epistemológica consiste en buscar las conexiones y las regularidades existentes a través de la construcción intelectiva de los denominados tipos ideales ${ }^{5}$, que son conceptos fundamentales de su propuesta sociológica.

De esta propuesta, la teoría organizacional ha rescatado sobre todo la teoría de la dominación. Weber considera que la dominación es «la probabilidad de hallar obediencia a un mandato determinado [que] puede fundarse en diversos motivos: [... intereses, [costumbres o afecto]» (1964: 706). Este tipo específico de acción social se desarrolla a través de un sistema de dos actores y dos acciones: un dominador, un dominado; un mandato, una obediencia.

En esta teoría existen tres tipos ideales posibles: la dominación carismática, la tradicional y la legal racional o burocrática. Si bien la existencia de estos tipos ideales no está planteada de manera explícita en términos evolutivos, lo cierto es que - teóricamente al menos - al principio solo hubo una, la primera, luego, aparecen los rasgos de la segunda, es decir, esta tiene espacios de convivencia con aquella. Cuando aparece históricamente la tercera con el surgimiento del Estadonación y la empresa-capitalista, esta se vuelve hegemónica en el mundo occidental, por lo que es posible encontrar situaciones o acciones de dominación que van a corresponder con las anteriores.

Recordemos que lo que distingue a una forma de dominación de otra es lo que hace posible su legitimación. En el caso de la dominación carismática, la fuente de legitimación serán los liderazgos, en el caso de la tradicional, la tradición, la gracia y el arbitrio, mientras que en la burocrática, será la racionalidad, expresada en estatutos, jerarquías y documentos.

A partir de lo anterior, como el tipo de dominación burocrática es el que caracteriza a la modernidad en la que vivimos, vamos a tener como eje de las reflexiones sobre el ser en Weber a este tipo ideal. En este caso, el tipo ideal de organización racional es la burocracia, producto tardío de la evolución que solo ha sido posible, como ya comentamos, con la aparición del Estado moderno en un contexto capitalista que busca legitimidad y dominio a partir del ejercicio racionalizado de la interacción social. Entonces, siguiendo el hilo argumental, el individuo está siendo siempre dominado - al menos potencialmente - aunque la fuente de dominación pueda ser de diferente tipo. En este sentido el hombre es un individuo que obedece las órdenes de otros, sea a través de la fuerza (el ejerci-

5 «Esta construcción presenta el carácter de una utopía, obtenida mediante el realce conceptual de ciertos elementos de la realidad. Su relación con los hechos empíricamente dados de la vida consiste exclusivamente en esto: allí donde en realidad se comprueba o se supone que en algún grado operan conexiones del tipo abstractamente representado en aquella construcción, [...] podemos ilustrar y volver comprensible pragmáticamente la especificación de tal conexión en un tipo ideal» (Weber, 1973: 79). 
cio del poder ${ }^{6}$ ) o a través del consenso (en el que se actúa con consentimiento y voluntad). Para Weber la burocracia es la forma técnica más eficiente de organización, aquí, el hombre como mano tayloriano se complejiza en la medida en que se establece de manera explícita que a lo que obedece el ser es a la razón expresada en los lineamientos y procesos burocráticos. De modo que el ser es ahora una mano con conciencia de la razón que lo guía, se transforma entonces en un hombre que obedece, que establece y sigue reglas/leyes/normas, aunque sigue siendo parte de una máquina que busca la eficiencia de la estructura. Como se ha dicho, las relaciones sociales son entendidas a través de la dominación, sin embargo, el hombre sigue desdibujado, atrapado - metafóricamente - por una estructura burocrática, pues a pesar de que para Weber es la mejor forma de organización social, esta tiene efectos no deseados, disfunciones, que en su momento histórico no logró ver.

Para Weber el foco para entender al individuo en las acciones organizadas son las formas de poder y la legitimidad (¿cómo se establecen y se obedecen las leyes?). En Weber es posible hablar de un hombre racional, simbólico, que actúa con intereses y fines influenciados por el grupo social al que pertenece. Un individuo que dentro de la estructura burocrática busca la eficiencia a través de sus acciones. Este sujeto puede actuar en comunidad (entendido como el actuar orientado hacia expectativas, un comportamiento que puede observarse históricamente o construirse teóricamente), actuar asociado (pacto racional entre medios y fines), actuar en consenso (consenso amorfo como el habla), actuar institucionalmente (definida la institución como un pacto con el grupo, es la parte racionalmente ordenada de una acción grupal). Aquí no existe un lugar claro para hablar del sujeto y sus emociones, aunque Weber (2004) concede un papel importante a la angustia en el ethos capitalista. En suma, el sujeto se mueve en relación con reglas, normas, rituales, símbolos que son todos culturales y, por lo tanto, colectivos.

De esta manera, y para resumir antes de pasar a comentar a nuestro siguiente clásico, podemos decir que el sujeto «moderno» de Weber está en el mismo extremo de la racionalidad de nuestros continuos de discusión, y por el otro lado, en el continuo de la determinación frente a la libertad. Ya que la condición que supone que la dominación requiera de la obediencia asume la posibilidad de que esto pueda no suceder así, lo que le otorga al individuo cierta libertad, por lo que el sujeto transita en cierta medida a la condición de actor o agente, y su posición en el continuo se mueve hacia la libertad en cierto grado.

6 «Significa la probabilidad de imponer la propia voluntad, dentro de una relación social, aun contra toda resistencia y cualquiera que sea el fundamento de esa probabilidad» (ibid.: 43). 


\subsection{Elton Mayo. Escuela de las relaciones humanas}

El trabajo solo puede realizarse en estado de equilibrio (Mayo, 1972: 40).

Con la contribución de Elton Mayo a los estudios organizacionales es posible hablar del surgimiento de la escuela de las relaciones humanas, ya que aparece un nuevo estilo de imaginar y estudiar la acción organizada. Las investigaciones que dirigió este psicólogo industrial norteamericano en la empresa Western Electric Company, que son conocidas como el experimento de Hawthorne, entre 1927 y 1932, permitieron la aparición del homo affectus, pues el foco de su interés era saber más sobre el aspecto humano en las condiciones laborales. Sus preocupaciones iniciales fueron la monotonía y la fatiga en el estado de ánimo de la persona. Desde esta perspectiva, lo más significativo era entender el funcionamiento del sujeto dentro de una organización, y no el funcionamiento y la eficiencia de la estructura como se pudo advertir en las escuelas anteriores. Sus experimentos duraban largos períodos de tiempo, algunos de ellos modificaban las condiciones laborales, tiempos, movimientos, incentivos, entre otros, es decir, elementos que le permitían estudiar cambios y efectos en los trabajadores. Sus estudios estaban basados en observación, pruebas fisiológicas (por ejemplo, medición de pulso cardíaco, medidas bioquímicas, por señalar algunas) y cuestionarios. Aunque durante el último período también incluyó entrevistas.

Uno de los mayores descubrimientos de Mayo fue la relevancia de la organización informal, esa que no aparece en la estructura normativa sino que se hace día a día a través de las relaciones y que está organizada, más que por la razón, por una red de emociones y valores que portan los actores, acción organizada que en la interacción adopta su condición propia de red. Es por eso que para este autor antes de mejorar las condiciones laborales es necesario saber qué piensan las personas que laboran ahí. Con sus estudios se dio cuenta de la estrecha relación existente entre la actitud y la eficacia de los trabajadores, así como de la necesidad de contar con sistemas adecuados de comunicación que faciliten las relaciones y moldeen de alguna manera los comportamientos de los individuos en ámbitos organizacionales.

La influencia de Mayo ha sido determinante en el campo de los estudios organizacionales, se le considera el padre de la escuela de las relaciones humanas debido a la atención que colocó en las emociones, con él sale a la luz una concepción antropológica que pone ante todo al ser, en cuanto ser intelectual y emocional, que debe ser tomado en cuenta dentro de los espacios sociales organizados. Parte del éxito de su planteamiento se debe a la inclusión de las entrevistas como fuentes de información para la investigación, ya que a partir de ellas pudo dar sentido a las emociones que vivían los protagonistas de la industria: los trabajadores. Fue 
así que comenzó a comprender con mayor agudeza la naturaleza y la dimensión de los problemas que se viven dentro de las organizaciones. Mayo brindó una propuesta epistemológica distinta que busca comprender al ser humano desde sus emociones. Sin embargo, como señala Illouz (2006: 41), «lo que tal vez resulte más interesante es el hecho de que en el experimento que Mayo llevó a cabo en General Electric todos los participantes fueron mujeres, por lo cual, y sin que él se lo propusiera, sus hallazgos tenían un fuerte sesgo de género», sobre esto se podría realizar un análisis, pero por ahora lo más importante es señalar que a partir de estos estudios apareció lo que se denomina hombre corazón en los estudios organizacionales.

Así, nuestro homo affectus será entonces un ser de valores antes que de razón, muy diferente al de los racionalistas Taylor y Weber. Con respecto al continuo de libertad frente a determinación, pensemos que el condicionamiento que este tenga en su comportamiento va a estar guiado por una condición que será estructural en la medida en que los valores o sentimientos lo motiven, y estarán elaborados culturalmente. Es decir, si pensamos que uno de los descubrimientos más populares de Mayo es el del efecto Hawnthorne, aquel que hace que la eficiencia mejore en la medida en que los individuos involucrados en proyectos colectivos y relevantes para la organización se sientan parte de esta realidad, entonces esa condición cultural lo aproxima al extremo de lo colectivo frente a lo individual, y, por lo tanto, al de la determinación.

\subsection{Herbert Simon. Teoría de la decisión y el comportamiento.}

El énfasis estará sobre la forma en que los límites de nuestra capacidad para calcular y conducirnos razonablemente imponen límites similares sobre la capacidad de nuestras instituciones (Simon, 1989: 3).

Un par de décadas después de que los estudios organizacionales se centraran en las emociones humanas, como en un movimiento pendular, se desarrolló una teoría desde la economía que hizo regresar el focus del pensamiento organizacional a la razón, solo que, esta vez, intentando superar algunas de las críticas que se habían hecho de manera importante al racionalismo que había inspirado a nuestros dos primeros autores como herederos de este «espíritu» a la economía neoclásica. El autor responsable de esta aproximación fue Herbert Simon, quien centró su atención en el hecho administrativo y los procesos decisorios involucrados en el mismo ${ }^{7}$. Su pensamiento y contribución han sido decisivos para entender cómo es que las acciones sociales son dirigidas por una racionalidad limitada y no por

7 «Las decisiones son algo más que proposiciones de hecho. Desde luego describen un estado futuro de cosas y esta descripción puede ser verdadera o falsa en un sentido estrictamente empírico; pero poseen, además, una 
una racionalidad total, como se había pensado hasta ese momento. Para este autor «la mejor manera de lograr una visión intensa de la estructura y del funcionamiento de una organización es analizar la manera en que las decisiones y el comportamiento de esos empleados se ven influidos dentro de y por la organización» (Simon, 1947: 4). En ese sentido, y como afirma el propio autor, todo proceso administrativo es un proceso de decisión ${ }^{8}$. Esta idea fundamental centrada en las decisiones humanas influyó en muchos campos del conocimiento, económicos, políticos y sociales, incluso hacia finales de su carrera, Simon comenzó a simular procesos de decisión a través de computadoras llevando así la tecnología a las ciencias sociales. El trabajo de toda una vida y su valiosa contribución al conocimiento le hicieron ganar en 1978 el premio Nobel de Economía.

Para este autor, la economía debe estudiar los asuntos cotidianos así como examinar la acción individual y social, ya que «el carácter del ser humano se moldea mucho más por el trabajo diario, los materiales y los recursos con los que tiene contacto que por cualquier otra influencia, al menos que ésta provenga de sus ideales religiosos» (Simon, 1978:343) ${ }^{9}$. Esto explica —en parte- por qué Simon se centró en estudiar el día a día de los procesos de decisión dentro de una organización, ya que con el análisis de lo cotidiano logró demostrar que no existe una racionalidad total en las personas, como suponían la teorías clásicas de la economía y la administración hasta ese momento, sino una racionalidad limitada (variable de sujeto a sujeto) por capacidades valores y conocimientos. Entonces, el individuo está limitado tanto por su capacidad, habilidades, hábitos y reflejos como por sus valores y sus conocimientos. Para Herbert Simon el carácter de un individuo es moldeado por los asuntos ordinarios de la vida, por el trabajo diario, por los recursos que tiene a su alcance y por los valores con los que ha ido creciendo; esto conforma todo el bagaje con el que habrá de elegir su camino y sus estrategias dentro de las organizaciones sociales.

Para Simon (1947), entonces, la racionalidad se ocupa de la elección de las alternativas preferidas que tiene a su disposición el actor social dentro de su actividad organizada. Esta elección surge de acuerdo con un sistema de valores cuyas consecuencias de comportamiento puedan ser valoradas. De esta manera, la racionalidad no se entiende separada de los valores $y$, por lo tanto, se consideran

cualidad imperativa: seleccionan un estado futuro de cosas con preferencia a otro y dirigen el comportamiento hacia la alternativa elegida. En una palabra: tienen un contenido ético tanto como fáctico» (Simon, 1947: 45).

8 «Cada decisión comprende la selección de una meta y un comportamiento relacionado con la misma; esta meta puede, a su vez, ser un medio para una finalidad algo más lejana, y así hasta que se llega a un objetivo relativamente último. Cuando las decisiones llevan a la selección de finalidades últimas, las llamaremos "juicios de valor"; cuando impliquen el logro de tales finalidades, las llamaremos "juicios de hecho"» (ibid.: 6).

9 Traducción propia del texto original en inglés titulado Rational Decisión-Making in Business Organizations (Simon, 1978). 
integrados en la acción de los actores. Lo mismo podemos decir con respecto a la noción de orden, ¿̇es el individuo y su libertad o lo colectivo estructurado y su condicionamiento lo que funda el orden social? Lo que intentamos argumentar aquí es que, de la misma manera que con respecto a los extremos de la explicación posible de la acción, en el caso del orden, Simon también va a ser un teórico integrador, y esta condición es la que lo vuelve un autor fundamental para cualquier aportación posterior sobre el pensamiento organizacional.

A continuación se sintetizan los ejes que sustentan esta racionalidad limitada de Simon (1947: 78). Primero, que la racionalidad total demanda un conocimiento completo de las consecuencias a cada elección, algo que en la vida real no sucede, puesto que el conocimiento de las consecuencias es siempre fragmentario. Segundo, dado que estas consecuencias pertenecen al futuro no es posible asignarles un valor, es por eso que el sujeto tiene que hacer uso de su imaginación y anticipar el valor de sus decisiones, pero esta asignación anticipada siempre será imperfecta. Tercero, que la racionalidad total de la que se habla en la teoría clásica exige que el sujeto escoja entre todos los posibles comportamientos alternativos, algo que en lo cotidiano no ocurre porque solo se conocen unas pocas alternativas posibles, por lo tanto, la racionalidad total es una falacia.

Según este autor es posible señalar diversos factores que intervienen en la racionalidad (limitada) del individuo, por un lado, la docilidad ${ }^{10}$, el papel de la memoria ${ }^{11}$ en el comportamiento, el hábito ${ }^{12}$, los estímulos positivos (estos estímulos pueden ser en parte razonados y en parte encarnados por el hábito), los determinantes del medio ambiente psicológico y los mecanismos de integración del comportamiento (en los que se pueden diferenciar mecanismos de persistencia o de iniciación del comportamiento). En concordancia con esto, los procesos de toma de decisión dentro de una organización pueden resumirse en dos, aquellos que han sido programados y aquellos que no fueron programados (que pueden ser problemas emergentes a resolver) en los cuales el motor que mueve a la decisión es la motivación y/o las motivaciones que pueden ser moldeadas/influidas tanto por el individuo como por el control de la organización y/o los factores externos a la misma. En este sentido, la autoridad ${ }^{13}$ y el

10 «Facultad del ser humano de observar las regularidades de tipo muy general y de comunicarlas a otros seres humanos» (Simon, 1947: 82).

11 «El papel de la memoria en el comportamiento racional apenas necesita comentario. Cuando se presentan problemas similares, la memoria se encarga de almacenar la información reunida, e incluso las conclusiones a que se ha llegado al resolver el primer problema y las pone a nuestra disposición, sin nueva investigación, cuando nos sale al paso el siguiente problema de la misma clase» (ibid.: 84).

12 «El hábito es un mecanismo igualmente importante que ayuda a conservar los modelos útiles de comportamiento. El hábito permite evitar el esfuerzo mental, eliminando el área del pensamiento consciente aquellos aspectos de la situación que se repiten con mucha frecuencia» (ibid.: 84).

13 «[Zona de aceptación]. Se dice que un subordinado acepta la autoridad siempre que consiente que su comportamiento sea guiado por la decisión de un superior, sin examinar libremente las razones de esa decisión. 
desarrollo de lealtades ${ }^{14}$ organizativas son dos de los medios más importantes para que la organización influya en las premisas valorativas del individuo.

Ahora bien, con respecto a la concepción antropológica de este autor, podemos decir que, para él, los seres humanos somos tomadores de decisiones de racionalidad limitada, idea que superó, por mucho, la visión del hombre como máquina en la que el énfasis estaba en los movimientos y en la satisfacción económica y/o de producción. Llevar la mirada a las motivaciones y las decisiones permitió entender a los individuos desde una teoría de la acción, una acción en la que el ser humano decide en estrecha relación con la organización. Consideramos que este enfoque no deja de ser económico y racional, pues a pesar de que afirma la existencia de una racionalidad limitada, su obra muestra que él estaba decidido a diseñar un modelo tecnológico, automatizado, que permitiera prever las decisiones de los individuos en un marco organizacional. Esto nos permite aventurar la idea de que este autor creía en la posibilidad de una racionalidad tecnológica y en el control técnico del ser dentro de las organizaciones. Sin embargo, como él mismo señala:

El comportamiento humano, incluso el comportamiento humano racional no puede ser contabilizado por un sinfín de invariantes, el comportamiento humano no se explica asumiendo una perfecta adaptación al entorno. Los mecanismos básicos del comportamiento humano pueden ser relativamente simples, sin embargo, la simplicidad funciona en interacción con condiciones impuestas por un medio ambiente extremadamente complejo, por la propia memoria humana a largo plazo y por la capacidad de aprender que tienen los seres humanos tanto individual como colectivamente (Simon, $1978: 368)^{15}$.

Con esta noción de que el comportamiento humano no puede ser contabilizado, queremos cerrar este apartado, para dejar clara la idea de que la racionalidad limitada no solo explica los procesos de decisión dentro de las organizaciones sino los procesos de intelección limitados que los seres humanos realizan cuando se acercan a la realidad social. Puesto que «en la ciencia empírica aspiramos sólo a verdades aproximadas» (ibid.: 366), el ser humano es y seguirá siendo un misterio del que solo podremos conocer un poco más.

\footnotetext{
El superior, cuando ejerce autoridad, no trata de convencer al subordinado, sino únicamente de conseguir su conformidad [...] la autoridad, en el sentido aquí definido, puede operar dentro de la organización "hacia arriba" y "hacia abajo" [...] las "líneas de autoridad" representadas en los organigramas tienen, sin embargo, una significación especial, ya que se recurre comúnmente a ella para poner fin a un debate cuando se comprueba que es imposible llegar a un acuerdo sobre una decisión determinada» (ibid.: 11).

14 «Una característica dominante del comportamiento humano es que los miembros de un grupo organizado tienden a identificarse con ese grupo. Al tomar decisiones, la lealtad organizativa les induce a valorar las vías alternativas de acción, en relación con las consecuencias que esta tendrá para el grupo [...] este fenómeno de identificación o lealtad a la organización desempeña en la administración una función muy importante» (ibid:: 14). 15 Traducción propia del texto en inglés.
} 


\section{Autonomía-determinación y/o razón-sentimientos, reflexiones finales}

Si bien la construcción que hacemos en este artículo sobre los criterios para la fundamentación del carácter de clásico en los autores revisados aquí será relevante solo para los estudiosos de la teoría organizacional, creemos — desde nuestra perspectiva - que lo más interesante y/o importante de esta propuesta será el haber desarrollado uno de los cuestionamientos centrales de la filosofía aplicada a la TO. Dicho de otra manera, poner en acción un cuestionamiento típicamente filosófico en la arena de las organizaciones. En este caso, tratar de responder qué es el ser humano en la realidad organizacional y qué características le atribuyen los autores clásicos de la TO.

$\mathrm{Al}$ respecto, al hacer los autores el recuento de lo escrito en este artículo, llegábamos a la conclusión de que cuando los autores clásicos de la TO hacen propuestas acerca de cómo lograr eficiencias o mejoras en las organizaciones en términos teóricos, necesariamente lo hacen desde una referencia lógica que surge de una postura antropológica, incluso cuando esta no haya sido expresada de manera explícita. Y es que cuando se habla de la realidad, necesariamente el sujeto ha de tener elaborada - consciente o inconscientemente- una idea sobre lo que es la realidad antropológicamente hablando, qué es el ser humano, cuáles sus acciones y sus formas de relacionarse. Asumimos que para teorizar sobre las acciones en una organización se tiene que tener primero - fundado en el conjunto de cuestionamientos a reflexionar/analizar - un supuesto antropológicoontológico sobre la realidad, sobre el ser. A esta posibilidad que relaciona la teoría con un fundamento antropológico la llamamos aquí congruencia lógica.

Pensamos, además, que la postura antropológica que los autores plantean o dejan dilucidar — en mayor o menor medida - en su pensamiento teórico, en sus ideas sobre la realidad humana organizacional, tiene implícita una posibilidad ética. Nos referimos a la posibilidad que se le otorga al individuo para moverse dentro de la estructuración social en la que vive. Es decir, solo si los seres humanos son concebidos como libres desde la postura teórica pueden tener abierta -en mayor mesura - una posibilidad ética de libertad en la acción organizacional. Pero no todos los autores otorgan el mismo grado de libertad al individuo. Solo los sujetos libres, con capacidad de discernir entre alternativas de acción, pueden realizar este juicio considerando lo bueno o mejor como un valor de referencia o criterio de elección. Para el desarrollo de este proceso en la realidad humana, hay dos posibles características presentes en los teóricos clásicos de la TO que van a permitir el juicio (de valor o de hecho): la razón y el valor o norma cultural. 
En este sentido entonces, resumiendo, lo que el lector pudo ver en este trabajo es la revisión analítica de las posturas antropológicas de los autores de la teoría organizacional clásica, su congruencia lógica, que está presente al integrar en el análisis las propuestas más programáticas de sus textos, y la posibilidad ética que tratamos de develar al reflexionar sobre los grados de libertad que los propios autores le otorgaban al ser humano a través de sus supuestos teóricos sobre las organizaciones.

En suma, podemos resumir estas posturas asumiendo, con respecto a la acción, que tanto Taylor como Weber ven la razón - la racionalidad- de los seres humanos como central en la estructuración posible. Es la razón la que puede explicar - de manera legítima - las interacciones sociales de los seres humanos en las organizaciones. De modo que los individuos han de ser racionales. Solo que de manera paradójica. Los seres han de estar determinados, en el primer caso, por el diseño de los procesos, y por la ciencia y sus métodos en el segundo. Habrá que enfatizar que en el caso de Weber el nivel de determinación va a estar dado por la obediencia requerida, expresada como legítima en el orden de la modernidad y por los productos de la razón expresados como características burocráticas. En el caso de Taylor esta obediencia está determinada por la racionalidad de la organización, es el modelo máquina en el que el hombre no aspira a ser nada más que una mano que trabaja.

Mayo, analizado desde un sentido histórico, aparece enseguida posicionándose en el otro extremo del continuo de la acción, asumiendo los valores (en este caso denominados sentimientos) como el criterio de explicación central de las acciones humanas, y asumiendo una congruencia lógica con la idea de que entonces el asunto del orden se expresa desde elementos culturales y, por lo tanto, colectivos. El argumento central yace en dos pilares: la cultura y el sentimiento. El grado de libertad que se le otorga al ser humano está indudablemente ligado a sus pasiones, a sus emociones, al modo en el que este puede librarse de lo que siente. $\mathrm{Al}$ modo en el que la propia cultura lo determina. Esto nos lleva a posicionarnos en una concepción antropológica que pone de manifiesto los elementos «ausentes», «ignorados» antes por la teoría organizacional. Sin embargo, nos pone nuevamente en el extremo, poniendo en la mesa de la reflexión a un ser puro corazón.

Simon, finalmente, es un autor que podemos entender como integrador, sumando a la razón el asunto de los valores, y asumiendo que toda decisión individual está mediada y condicionada por elementos del contexto que son en sí mismo colectivos.

Es quizá este elemento integrador el que le falta por reconocer a Simon, y que ayudaría a explicar la relevancia que aún tiene en esta y en otras tantas disciplinas. 


\section{Bibliografía}

Ahumada Figueroa, Luis (2001). Teoría y cambio en las organizaciones. Un acercamiento desde los modelos de aprendizaje organizacional. Valparaíso: Ediciones universitarias de Valparaíso-UCV.

Alexander, Jeffrey C. (2008). Las teorías sociológicas desde la Segunda Guerra Mundial. Barcelona: Gedisa.

Alonso Munguía, Enrique y Ocegueda Melgoza, Vicente (2006). Teoría de las organizaciones. Zapopan: Editorial Umbral.

Argyris, Chris (1957). Personality and Organization. New York: Harper Collins.

Barnard, Chester (1968). The Functions of the Executive. Cambridge: Harvard University.

Compagnon, Antoine y Carol Cosman (2004). Literature, Theory and Common Sense. Princeton; Oxford: Princeton University Press Google Scholar.

Crozier, Michel y Friedberg, Erhard (1989). El actor y su estrategia. México: Alianza Editorial Mexicana.

Dávila L. De Guevara, Carlos (2001). Teorías organizacionales y administración. Enfoque crítico. Bogotá: McGraw-Hill.

Gadamer, Hans-Georg (1999). Verdad y método I. Salamanca: Sígueme.

Ibarra Colado, Eduardo (1999). «Los saberes sobre la organización: etapas, enfoques y dilemas». En C. Castillo Mendoza (coord.). Economía, organización y trabajo: un enfoque sociológico (pp. 95-154). Madrid: Pirámide.

Illouz, E. (2006). Intimidades congeladas: las emociones en el capitalismo. Buenos Aires: Katz.

Jauss, Hans Robert (2013). La historia de la literatura como provocación. Madrid: Gredos.

March, James y Simon, Herbert (1980). Teoría de la organización. Barcelona: Ariel.

Mayo, Elton (1972). Problemas humanos de una civilización industrial. Buenos Aires: Nueva visión.

Pariente, José Luis (2001). Teoría de las organizaciones. Un enfoque de metáforas. México: Miguel Ángel Porrúa.

Piñeres, Juan David (2011). «Aproximaciones al primer debate sobre Bentham en Colombia: concepciones antropológicas, disputas educativas, aspiraciones nacionales». Revista de estudios sociales, (39), 11-23.

Pugh, Derek y Hickson, David J. (2007). Great writters in organizations. London: Ashgate. 
Ramió, Carles (2010). Teoría de la organización y administración pública. Madrid: Tecnos.

Ramió, Carles y Xavier Ballart (1992). Lecturas de teoría de la organización. Vol. 1. La evolución bistórica del pensamiento organizativo. Los principales paradigmas teóricos. Madrid: MAP.

Rivas, Luis Arturo (2009). «Evolución de la teoría de la organización». Revista Universidad E Empresa, Universidad del Rosario, 17: 11-32.

Shafritz, Jay. M;; Otт, J. Steven y Jang, Yong Suk (2015). Classics of organization theory. Cengage Learning ( $8{ }^{a}$ ed.).

Simon, Herbert (1947). El comportamiento administrativo. México: Aguilar

Simon, Herbert (1978). Rational Decision-Making in Business Organizations. Nobel Memorial Lecture. Pittsburgh: Carnegie-Mellon University.

Simon, Herbert (1989). Naturaleza y límites de la razón bumana. México: Fondo de Cultura Económica.

TAYlor, Frederick Winslow (1967). Principios de la administración científica. México: Herrero Hermanos.

Weber, Max (1964).Economía y sociedad. México: Fondo de Cultura Económica Weber, Max (1973). «Sobre algunas categorías de la sociología comprensiva». En M. Weber. Ensayos sobre metodología sociológica (pp. 175-221). Buenos Aires: Amorrortu.

Weber, Max (2004). La ética protestante y el espíritu del capitalismo. México: FCE. 